\title{
GANTMACHER-KREǏN THEOREM FOR 2 NONNEGATIVE OPERATORS IN SPACES OF FUNCTIONS
}

\author{
O. Y. KUSHEL AND P. P. ZABREIKO
}

Received 26 June 2005; Accepted 1 July 2005

The existence of the second (according to the module) eigenvalue $\lambda_{2}$ of a completely continuous nonnegative operator $A$ is proved under the conditions that $A$ acts in the space $L_{p}(\Omega)$ or $C(\Omega)$ and its exterior square $A \wedge A$ is also nonnegative. For the case when the operators $A$ and $A \wedge A$ are indecomposable, the simplicity of the first and second eigenvalues is proved, and the interrelation between the indices of imprimitivity of $A$ and $A \wedge A$ is examined. For the case when $A$ and $A \wedge A$ are primitive, the difference (according to the module) of $\lambda_{1}$ and $\lambda_{2}$ from each other and from other eigenvalues is proved.

Copyright (c) 2006 O. Y. Kushel and P. P. Zabreiko. This is an open access article distributed under the Creative Commons Attribution License, which permits unrestricted use, distribution, and reproduction in any medium, provided the original work is properly cited.

\section{Introduction}

In the monograph [3] the following statement was proved: if the matrix A of a linear operator $A$ in the space $\mathbb{R}^{n}$ is primitive along with its associated $\mathbf{A}^{(j)}(1<j \leq k)$ up to the order $k$, then the operator $A$ has $k$ positive simple eigenvalues $0<\lambda_{k}<\cdots<\lambda_{2}<\lambda_{1}$, with a positive eigenvector $e_{1}$ corresponding to the maximal eigenvalue $\lambda_{1}$, and an eigenvector $e_{j}$, which has exactly $j-1$ changes of sign, corresponding to $j$ th eigenvalue $\lambda_{j}$ (see [3, page 310, Theorem 9]). Matrices with mentioned features are called henceforth $k$-completely nonnegative; in the most important case $k=n$ they are called oscillatory.

Naturally, there arises a problem whether it is possible to extend this statement to operators in infinite-dimensional spaces, for example, to linear integral operators. This problem practically has not been studied in full volume. However, in the monograph [3], Gantmacher and Kreĭn have thoroughly studied the linear integral operators

$$
K x(t)=\int_{a}^{b} k(t, s) x(s) d s
$$

Hindawi Publishing Corporation

Abstract and Applied Analysis

Volume 2006, Article ID 48132, Pages 1-15

DOI 10.1155/AAA/2006/48132 
acting in the space $L_{2}([a, b])$ with continuous kernels $k(t, s)$, for which the matrices $\left\|k\left(t_{i}, t_{j}\right)\right\|_{1}^{n}(n=1,2, \ldots)$ for any points $t_{1}, \ldots, t_{n} \in[a, b]$, among which at least one is interior, are oscillatory. Such kernels, named in [3] oscillatory, form quite full analogue to oscillatory matrices. In [3], for the integral operators with continuous oscillatory kernels, it was proved that there exists a converging-to-zero sequence of positive simple eigenvalues $\lambda_{1}>\lambda_{2}>\cdots>\lambda_{n}>\cdots$ with eigenfunctions $e_{n}(t)$ that has exactly $n-1$ changes of sign, corresponding to the $n$th eigenvalue $\lambda_{n}$ (see [3, page 211]).

In connection with the formulated Gantmacher-Kreln theorem, there arises a natural question on the possibility of spreading the statements about $k$-completely-nonnegative matrices from [3] onto the integral operators with $k$-completely-nonnegative kernels, that is, the kernels $k(t, s)$, for which the matrices $\left\|k\left(t_{i}, t_{j}\right)\right\|_{1}^{n}(n=1,2, \ldots, k)$ for any points $t_{1}, \ldots, t_{n} \in[a, b]$, among which at least one is interior, are oscillatory. The answer to this question is positive. Moreover, this statement was actually proved exactly in [3].

However, here arises a question how substantial the condition of continuity of the kernel $k(t, s)$ is in these statements and how substantial the assumption that the problem is regarded in the space of functions, defined exactly on the interval $[a, b]$, is. And of course the natural question arises whether it is possible to obtain similar statements for abstract (not necessarily integral) operators in an arbitrary Banach spaces.

In the present paper we study 2-completely-nonnegative (or otherwise bi-nonnegative) operators in the spaces $L_{p}(\Omega)(1 \leq p \leq \infty)$ and $C(\Omega)$. As the authors believe, the natural machinery for the examination of such operators is a crossway from studying an operator $A$ in one of the spaces $L_{p}(\Omega)$ and $C(\Omega)$ to the study of the operators $A \otimes A$ and $A \wedge A$, acting, respectively, in the spaces $L_{p} \otimes L_{p}=L_{p}(\Omega \times \Omega)$ and $L_{p} \wedge L_{p}=L_{p}^{a}(\Omega \times \Omega)$ (the latter is a subspace of the space $L_{p} \otimes L_{p}=L_{p}(\Omega \times \Omega)$, consisting of antisymmetric functions, i.e., functions $x(t, s)$, for which $x(t, s)=-x(s, t))$.

\section{Tensor and exterior square of the spaces $L_{p}(\Omega)$ and $C(\Omega)$}

Let $(\Omega, \mathfrak{A}, \mu)$ be a triple consisting of some set $\Omega$, some $\sigma$-algebra $\mathfrak{A}$ of "measurable" subsets and some $\sigma$-finite and $\sigma$-additive measure on $\mathfrak{A}$. We will be interested in the space $L_{p}(\Omega)$ of functions, integrable on $\Omega$ with the power $p$ for $1 \leq p<\infty$ or measurable and substantially bounded for $p=\infty$, the analogous space $L_{p}(\Omega \times \Omega)$ of functions, integrable on $\Omega \times \Omega$ with the power $p$ for $1 \leq p<\infty$ or essentially bounded for $p=\infty$ and, finally, the subspace $L_{p}^{a}(\Omega \times \Omega)$ of the space $L_{p}(\Omega \times \Omega)$ of antisymmetric functions. Henceforth let $p$ be a fixed number from $[1, \infty]$.

We start with observing the following facts:

(1) the space $L_{p}^{a}(\Omega \times \Omega)$ is one of the tensor products of the space $L_{p}(\Omega)$ by itself, and, respectively,

(2) the space $L_{p}^{a}(\Omega \times \Omega)$ is one of the exterior products of the space $L_{p}(\Omega)$ by itself.

The first of these statements means the following.

(a) For arbitrary functions $x_{1}, x_{2} \in L_{p}(\Omega)$ their $\odot$-product $x_{1} \odot x_{2}\left(t_{1}, t_{2}\right)=x_{1}\left(t_{1}\right) x_{2}\left(t_{2}\right)$ belongs to the space $L_{p}(\Omega \times \Omega)$, with

$$
\left\|x_{1}\left(t_{1}\right) x_{2}\left(t_{2}\right)\right\|=\left\|x_{1}\left(t_{1}\right)\right\|\left\|x_{2}\left(t_{2}\right)\right\|
$$


(b) The linear hull of the set of all $\odot$-products of functions from $L_{p}(\Omega)$, that is, the set of all functions of the form

$$
x\left(t_{1}, t_{2}\right)=\sum_{i} x_{1}^{i}\left(t_{1}\right) x_{2}^{i}\left(t_{2}\right)
$$

is dense in the space $L_{p}(\Omega \times \Omega)$.

The second statement means the following.

(a) The $\wedge$-product of arbitrary functions $x_{1}, x_{2} \in L_{p}(\Omega)$ with $x_{1} \wedge x_{2}\left(t_{1}, t_{2}\right)=$ $x_{1}\left(t_{1}\right) x_{2}\left(t_{2}\right)-x_{1}\left(t_{2}\right) x_{2}\left(t_{1}\right)$ also belongs to the space $L_{p}(\Omega \times \Omega)$, and it is obvious that

$$
\begin{gathered}
x_{1} \wedge x_{2}\left(t_{1}, t_{2}\right)=-x_{1} \wedge x_{2}\left(t_{2}, t_{1}\right), \\
\left\|x_{1} \wedge x_{2}\left(t_{1}, t_{2}\right)\right\| \leq 2\left\|x_{1}\left(t_{1}\right)\right\|\left\|x_{2}\left(t_{2}\right)\right\| .
\end{gathered}
$$

(b) The linear hull of the set of all $\wedge$-products of the functions from $L_{p}(\Omega)$ is dense in the space $L_{p}^{a}(\Omega \times \Omega)$.

The space $L_{p}^{a}(\Omega \times \Omega)$ is isomorphic in the category of Banach spaces to the space $L_{p}(W)$, where $W$ is the subset $\Omega \times \Omega$, for which the sets $W \cap \widetilde{W}$ and $(\Omega \times \Omega) \backslash(W \cup \widetilde{W})$ have zero measure; here $\widetilde{W}=\left\{\left(t_{2}, t_{1}\right):\left(t_{1}, t_{2}\right) \in W\right\}$ (such sets do always exist). Really, extending the functions from $L_{p}(W)$ as antisymmetric functions from $W$ to $\Omega \times \Omega$, we obtain the set of all the functions from $L_{p}^{a}(\Omega \times \Omega)$. Further, setting the norm of a function in $L_{p}(W)$ to be equal to the norm of its extension, we get that the spaces $L_{p}^{a}(\Omega \times \Omega)$ and $L_{p}(W)$ are isomorphic in the category of normed spaces.

The general scheme of the interrelations between the spaces $L_{p}(\Omega) \oplus L_{p}(\Omega), L_{p}(\Omega \times$ $\Omega), L_{p}^{a}(\Omega \times \Omega)$, and $L_{p}(W)$ can be represented by the diagram

$$
L_{p}(\Omega) \otimes L_{p}(\Omega) \stackrel{\otimes}{\longrightarrow} L_{p}(\Omega \times \Omega) \stackrel{\mathbf{a}}{\longrightarrow} L_{p}^{a}(\Omega \times \Omega)=L_{p}(W)
$$

where $\mathbf{a}$ is the antisymmetrization operator acting from $L_{p}(\Omega \times \Omega)$ to $L_{p}^{a}(\Omega \times \Omega)$ according to the rule

$$
\mathbf{a} x\left(t_{1}, t_{2}\right)=\frac{x\left(t_{1}, t_{2}\right)-x\left(t_{2}, t_{1}\right)}{2} .
$$

Let us examine some examples of constructing the set $W$ for different sets $\Omega$.

(1) Let $\Omega=[a, b]$; then $\Omega \times \Omega=[a, b]^{2}$, and as $W$ we may regard the triangle, defined by inequalities $a \leq t_{1} \leq t_{2} \leq b$. Really, in this case $\widetilde{W}$ is defined by the inequalities $a \leq t_{2} \leq t_{1} \leq b, \Omega \times \Omega=[a, b]^{2}=W \cup \widetilde{W}$ and $W \cap \widetilde{W}=w_{0}$, where $w_{0}$, defined by the inequalities $a \leq t_{1}=t_{2} \leq b$, is a set of zero measure.

(2) Consider another example. Let $\Omega=[a, b]^{2}$. Then $\Omega \times \Omega=[a, b]^{4}$. Define on the space $\mathbb{R}^{2}$ the following order relation: $\left(t_{1}, t_{2}\right) \leq\left(s_{1}, s_{2}\right)$, if $t_{1} \leq s_{1}$. Introduce $W=\left\{\left(t_{1}, t_{2}, t_{3}, t_{4}\right) \in[a, b]^{4}:\left(t_{1}, t_{2}\right)<\left(t_{3}, t_{4}\right)\right\}$ and $\widetilde{W}=\left\{\left(t_{1}, t_{2}, t_{3}, t_{4}\right) \in[a, b]^{4}:\right.$ $\left.\left(t_{3}, t_{4}\right)<\left(t_{1}, t_{2}\right)\right\}$. As we see, $\Omega \times \Omega=W \cup \widetilde{W} \cup w_{0}$, where $w_{0}=\left\{\left(t_{1}, t_{2}, t_{3}, t_{4}\right) \in\right.$ $\left.[a, b]^{4}:\left(t_{1}, t_{2}\right)=\left(t_{3}, t_{4}\right)\right\}$ is a set of zero measure in the 4-dimensional space. After thorough examination of the inequalities defining the set $W$, one obtains 
$W=\left\{a \leq t_{1}<t_{3} \leq b ; a \leq t_{2}, t_{4} \leq b\right\}$. The geometrical interpretation of this construction is as follows: the 4-dimensional cube is divided by the hyperflat $t_{1}=t_{3}$ into two symmetric parts. Notice that the cube can be divided in such a way with the help of another surfaces as well, for example, with the help of the hyperflat $t_{1}+t_{2}=t_{3}+t_{4}$.

(3) Suppose that on the set $\Omega$ a relation with the following properties is given:

(a) almost all the elements of $\Omega$ are comparable;

(b) $\mu\left(\left\{t_{1}, t_{2} \in \Omega: t_{1} \preceq t_{2}\right\} \cap\left\{t_{1}, t_{2} \in \Omega: t_{2} \preceq t_{1}\right\}\right)=0$. Then we can define the sets $W=\left\{\left(t_{1}, t_{2}\right) \in \Omega^{2}: t_{1} \preceq t_{2}\right\}$ and $\widetilde{W}=\left\{\left(t_{1}, t_{2}\right) \in \Omega^{2}: t_{2} \preceq t_{1}\right\}$, possessing the necessary properties.

Now let $(\Omega, \tau)$ be some compact Hausdorf topological space and let $C(\Omega)$ be the space of all continuous functions on $\Omega$. Then the set $\Omega \times \Omega$, with the topology $\tau \times \tau$ given on it, is also a compact Hausdorf space. Denote by $C(\Omega \times \Omega)$ the space of all continuous functions on $\Omega \times \Omega$, and by $C^{a}(\Omega \times \Omega)$ - the subspace $C(\Omega \times \Omega)$ of all antisymmetric functions on $\Omega \times \Omega$. Just as in the case of Lebesgue spaces, the space $C(\Omega \times \Omega)$ is one of the tensor products of the space $C(\Omega)$ by itself, and the space $C^{a}(\Omega \times \Omega)$ is one of the exterior products of the space $C(\Omega)$ by itself. In other words, for them the analogues of statements (1) and (2) for Lebesgue spaces are true. Further, the space $C^{a}(\Omega \times \Omega)$ is isomorphic to the space $C_{0}(W)$ (here $W$ is a subset $\Omega \times \Omega$, for which $W \cap \widetilde{W}=\Delta, \Delta=$ $\{(t, t): t \in \Omega\}, W \cup \widetilde{W}=\Omega \times \Omega$, and $C_{0}(W)$ is a subspace of the space $C(W)$, consisting of all functions $x\left(t_{1}, t_{2}\right)$, for which $\left.x\left(t_{1}, t_{1}\right)=0\right)$. In particular, the following diagram is true:

$$
C(\Omega) \otimes C(\Omega) \stackrel{\otimes}{\longrightarrow} C(\Omega \times \Omega) \stackrel{\mathbf{a}}{\longrightarrow} C^{a}(\Omega \times \Omega)=C_{0}(W) \subset C(W) .
$$

Sometimes Lebesgue spaces and the space of all continuous functions have to be examined at the same time. In this case it is natural to require continuous functions to be measurable. This means that the topology $\tau, \sigma$-algebra $\mathfrak{A}$, and the measure $\mu$ on $\Omega$ must be related to each other in the following way: $\mathfrak{A}$ contains all the closed sets from $\tau$ and the measure $\mu$ is regular, that is, for any $A \in \mathfrak{A}$ and any number $\epsilon>0$ there exist a closed set $F$ and an open set $G$ such that $F \subset A \subset G$ and $\mu(G \backslash F)<\epsilon$. In this case the space $C(\Omega)$ is associated with a closed subspace of the space $L_{\infty}(\Omega)$.

\section{Tensor and exterior squares of linear operators in the spaces $L_{p}(\Omega)$ and $C(\Omega)$}

Let $A$ and $B$ be continuous linear operators acting in the space $L_{p}(\Omega)$. These operators generate the operator $A \otimes B$ in the space $L_{p}(\Omega \times \Omega)$ as follows: on degenerate functions it is defined by the equality

$$
(A \otimes B) x\left(t_{1}, t_{2}\right)=\sum_{j} A x_{1}^{j}\left(t_{1}\right) \cdot B x_{2}^{j}\left(t_{2}\right) \quad\left(x\left(t_{1}, t_{2}\right)=\sum_{j} x_{1}^{j}\left(t_{1}\right) \cdot x_{2}^{j}\left(t_{2}\right)\right),
$$

and on arbitrary functions it is defined by extension via continuity from the subspace of degenerate functions onto the whole of $L_{p}(\Omega \times \Omega)$. The possibility of such an extension is due to the density of the set of all degenerate functions in the space $L_{p}(\Omega \times \Omega)$ and to 
the fact that the operator $A \otimes B$ is bounded on the subspace of degenerate functions of the space $L_{p}(\Omega \times \Omega)$; the latter comes out from the following observations.

Let

$$
x\left(t_{1}, t_{2}\right)=\sum_{j} x_{1}^{j}\left(t_{1}\right) x_{2}^{j}\left(t_{2}\right)
$$

be a degenerate function from $L_{p}(\Omega \times \Omega)$. It is obvious that for almost every $t_{2} \in \Omega$ this function is measurable as a function of $t_{1} \in \Omega$. Moreover, it can be regarded as a linear combination of functions $x_{1}^{j}\left(t_{1}\right)$ with coefficients $x_{2}^{j}\left(t_{2}\right)$. Therefore

$$
A_{(1)} x\left(t_{1}, t_{2}\right)=\sum_{j} A x_{1}^{j}\left(t_{1}\right) \cdot x_{2}^{j}\left(t_{2}\right)
$$

the obtained function turns out to be measurable with respect to all the variables (the operator's $A$ index (1) means that it is used for the variable $t_{1}$ ). Because of the Fubini theorem, the following estimate is true:

$$
\left\|A_{(1)} x\left(t_{1}, t_{2}\right)\right\| \leq\|A\|\left\|\sum_{j} x_{1}^{j}\left(t_{1}\right) \cdot x_{2}^{j}\left(t_{2}\right)\right\| .
$$

Further, the function $\sum_{j} A x_{1}^{j}\left(t_{1}\right) x_{2}^{j}\left(t_{2}\right)$ is measurable with respect to all the variables, it is measurable as a function of $t_{2} \in \Omega$ for almost every $t_{1} \in \Omega$, and it can also be regarded as a linear combination of functions $x_{2}^{j}\left(t_{2}\right)$ with coefficients $A x_{1}^{j}\left(t_{1}\right)$. Therefore, after using the operator $B$ for the variable $t_{2}$,

$$
B_{(2)} A_{(1)} x\left(t_{1}, t_{2}\right)=\sum_{j} A x_{1}^{j}\left(t_{1}\right) \cdot B x_{2}^{j}\left(t_{2}\right) ;
$$

the obtained function turns out to be measurable with respect to all the variables. Applying the Fubini theorem again, we get the estimate

$$
\left\|B_{(2)} A_{(1)} x\left(t_{1}, t_{2}\right)\right\| \leq\|A\|\|B\|\left\|\sum_{j} x_{1}^{j}\left(t_{1}\right) \cdot x_{2}^{j}\left(t_{2}\right)\right\| .
$$

We may conventionally write down the value of the operator $A \otimes B$ in the form of

$$
(A \otimes B) x\left(t_{1}, t_{2}\right)=A_{(1)} B_{(2)} x\left(t_{1}, t_{2}\right)=B_{(2)} A_{(1)} x\left(t_{1}, t_{2}\right),
$$

for arbitrary functions from $L_{p}(\Omega \times \Omega)$ as well. However, with such a separate usage of the operators $A$ and $B$, there arises a question of measurability of the function $B_{(2)} x\left(t_{1}, t_{2}\right)$ for the variable $t_{1}$. To avoid this trouble, we have to use the procedure of extension by continuity from the subspace of degenerate functions, where, as shown above, the mentioned trouble does not arise.

In the case of the space $C(\Omega \times \Omega)$, formula (3.7) and the estimate

$$
\left\|(A \otimes B) x\left(t_{1}, t_{2}\right)\right\| \leq\|A\|\|B\|\left\|x\left(t_{1}, t_{2}\right)\right\|
$$


arising from it are obvious, since the function that is continuous with respect to all the variables is continuous also in each variable separately with the fixed another one.

Further in this work we will examine exclusively the tensor square $A \otimes A$ of the operator $A$.

Let us examine the operator $A \wedge A: L_{p}^{a}(\Omega \times \Omega) \rightarrow L_{p}^{a}(\Omega \times \Omega)$, defined as the restriction of the operator $A \otimes A$ onto the subspace $L_{p}^{a}(\Omega \times \Omega)$. It is obvious that for degenerate antisymmetric functions the operator $A \wedge A$ can be defined by the equality

$$
(A \wedge A) x\left(t_{1}, t_{2}\right)=\sum_{j} A x_{1}^{j}\left(t_{1}\right) \wedge A x_{2}^{j}\left(t_{2}\right), \quad x\left(t_{1}, t_{2}\right)=\sum_{j} x_{1}^{j}\left(t_{1}\right) \wedge x_{2}^{j}\left(t_{2}\right) .
$$

Decompose $L_{p}(\Omega \times \Omega)$ into the direct sum of subspaces invariant with respect to $A \otimes A$ :

$$
L_{p}(\Omega \times \Omega)=L_{p}^{a}(\Omega \times \Omega) \oplus L_{p}^{s}(\Omega \times \Omega),
$$

where $L_{p}^{s}(\Omega \times \Omega)$ is the subspace of all symmetric functions from $L_{p}(\Omega \times \Omega)$. The operator $A \otimes A$ can be represented in the block form

$$
A \otimes A=\left(\begin{array}{cc}
A \wedge A & 0 \\
0 & \left.(A \otimes A)\right|_{L_{p}^{s}} ^{s}
\end{array}\right) .
$$

Further, it will be useful to compare the operator $A$ with the antisymmetrization of its tensor square $\mathbf{a} \circ(A \otimes A): L_{p}(\Omega \times \Omega) \rightarrow L_{p}^{a}(\Omega \times \Omega) \subset L_{p}(\Omega \times \Omega)$, where a is the antisymmetrization operator, defined by formula (2.5). Taking into account that the antisymmetrization operator leaves antisymmetric functions without changes, we conclude that the restriction of $\mathbf{a} \circ(A \otimes A)$ onto the subspace $L_{p}^{a}(\Omega \times \Omega)$ coincides with $A \wedge A$.

The space $C(\Omega \times \Omega)$ can also be decomposed into the direct sum of subspaces invariant with respect to $A \otimes A$ :

$$
C(\Omega \times \Omega)=C^{a}(\Omega \times \Omega) \oplus C^{s}(\Omega \times \Omega),
$$

where $C^{s}(\Omega \times \Omega)$ is the subspace of all symmetric functions from $C(\Omega \times \Omega)$. It is easy to see that the operator $A \otimes A: C(\Omega \times \Omega) \rightarrow C(\Omega \times \Omega)$ can also be represented in the block form. The operators $A \wedge A: C^{a}(\Omega \times \Omega) \rightarrow C^{a}(\Omega \times \Omega)$ and $\mathbf{a} \circ(A \otimes A): C(\Omega \times \Omega) \rightarrow$ $C^{a}(\Omega \times \Omega)$ are defined in the same way.

\section{Spectrum of the tensor square of linear operators in the spaces $L_{p}(\Omega)$ and $C(\Omega)$}

As usual, we will denote by $\sigma(A)$ the spectrum of the operator $A$, and by $\sigma_{p}(A)$ the point spectrum, that is, the set of all eigenvalues of the operator $A$. We will denote by $\sigma_{e b}(A)$ the Browder essential spectrum of the operator $A$, that is, the set of all points $\lambda \in \sigma(A)$, such that at least one of the following conditions holds:

(1) $R(A-\lambda I)$ is not closed;

(2) $\lambda$ is a limit point of $\sigma(A)$;

(3) $\cup_{n \geq 0} \operatorname{ker}(A-\lambda I)^{n}$ is of infinite dimension.

Thus $\sigma(A) \backslash \sigma_{e b}(A)$ will be the set of all isolated finite-dimensional eigenvalues of the operator $A$, (for more detailed information see $[6,7]$ ). 
In the papers by Ichinose [4-7] there have been obtained the results, representing the spectra and the parts of the spectra of the tensor product of linear bounded operators in terms of the spectra and parts of the spectra of the given operators under the natural suppositions that

(a) the tensor product of linear bounded operators $A \otimes B$ can be extended from the set of degenerate functions, and the extension is also a linear bounded operator in $L_{p}(\Omega \times \Omega)$ and $C(\Omega \times \Omega)$ respectively;

(b) the adjoint spaces $L_{p^{\prime}}(\Omega \times \Omega)$ and $r c a(\Omega \times \Omega)$ have the same property.

In fact these statements have been proved in the previous part. The explicit formulae, expressing the set of all isolated finite dimensional eigenvalues and the Browder essential spectrum of the operator $A \otimes A$ in terms of the parts of the spectrum of the given operator are obtained, for example, in [4, page 110, Theorem 4.2]. In particular, Ichinose proved, that for the tensor square of a linear bounded operator $A$ the following equalities hold:

$$
\begin{gathered}
\sigma(A \otimes A)=\sigma(A) \sigma(A) ; \\
\sigma(A \otimes A) \backslash \sigma_{e b}(A \otimes A)=\left(\sigma(A) \backslash \sigma_{e b}(A)\right)\left(\sigma(A) \backslash \sigma_{e b}(A)\right) \backslash\left(\sigma_{e b}(A) \sigma(A)\right) ; \\
\sigma_{e b}(A \otimes A)=\sigma_{e b}(A) \sigma(A) .
\end{gathered}
$$

Besides, for an arbitrary $\lambda \in\left(\sigma(A \otimes A) \backslash \sigma_{e b}(A \otimes A)\right)$ the following equality holds:

$$
\operatorname{ker}(A \otimes A-\lambda I \otimes I)=\operatorname{ker}\left(A-\lambda_{i} I\right) \otimes \operatorname{ker}\left(A-\lambda_{j} I\right),
$$

where $\lambda_{i}, \lambda_{j} \in\left(\sigma(A) \backslash \sigma_{e b}(A)\right)$ such that $\lambda=\lambda_{i} \cdot \lambda_{j}$.

In the finite-dimensional case, when the matrix $A \otimes A$ appears to be a tensor square of the matrix $A$, Stephanos's result ([13], see also [10]) tells that the set of all eigenvalues of the operator $A \otimes A$ is the set of all the possible products of the form $\left\{\lambda_{i} \lambda_{j}\right\}$, where $\left\{\lambda_{i}\right\}$ is the set of all eigenvalues of the operator $A$, repeated according to multiplicity. Thus the property

$$
\sigma_{p}(A \otimes A)=\sigma_{p}(A) \sigma_{p}(A)
$$

is widely known. In the infinite-dimensional case the analogous formula, expressing $\sigma_{p}(A \otimes A)$ in terms of the parts of the spectrum of the operator $A$, seems to be unknown. That is why further we will be interested in the case of a completely continuous operator A. For a completely continuous operator the following equalities are true:

$$
\left(\sigma(A) \backslash \sigma_{e b}(A)\right) \backslash\{0\}=\sigma_{p}(A) \backslash\{0\} ; \quad \sigma_{e b}(A)=\{0\} \text { or } \varnothing .
$$

So, from (4.2) we can get the complete information about the nonzero eigenvalues of the tensor square of a completely continuous operator:

$$
\sigma_{p}(A \otimes A) \backslash\{0\}=\sigma_{p}(A) \sigma_{p}(A) \backslash\{0\} .
$$

Here zero can be either a finite- or infinite-dimensional eigenvalue of $A \otimes A$, or a point of the essential spectrum. That is why, even for the case of a completely continuous operator, formula (4.4) in general is incorrect. 


\section{Spectrum of the exterior square of linear operators in the spaces $L_{p}(\Omega)$ and $C(\Omega)$}

For the exterior square, which is the restriction of the tensor square, the following inclusions are true:

$$
\begin{gathered}
\sigma(A \wedge A) \subset \sigma(A \otimes A) ; \\
\sigma_{p}(A \wedge A) \subset \sigma_{p}(A \otimes A) .
\end{gathered}
$$

In the finite-dimensional case, it is known that the matrix $A \wedge A$ in a suitable basis appears to be the second-order associated matrix to the matrix $A$, and we conclude that all the possible products of the type $\left\{\lambda_{i} \lambda_{j}\right\}$, where $i<j$, form the set of all eigenvalues of the exterior square $A \wedge A$, repeated according to multiplicity (see [3, Theorem 23, page 80]).

In the infinite-dimensional case we can also obtain some information concerning eigenvalues of the exterior square of a linear bounded operator.

Theorem 5.1. Let $X$ be either $L_{p}(\Omega)$ or $C(\Omega)$ and let $\left\{\lambda_{i}\right\}$ be the set of all eigenvalues of the operator $A: X \rightarrow X$, repeated according to multiplicity. Then all the possible products of the form $\left\{\lambda_{i} \lambda_{j}\right\}$, where $i<j$, will be the eigenvalues of the exterior square $A \wedge A$.

Proof. Let $\lambda_{i}, \lambda_{j} \in \sigma_{p}(A)$. Then there exist functions $x(t), y(t)$ from $X$, such that $(A-$ $\left.\lambda_{i} I\right) x(t)=0$ and $\left(A-\lambda_{j} I\right) y(t)=0$. Let us examine the value of the operator $A \wedge A-$ $\lambda_{i} \lambda_{j} I \wedge I$ on the degenerate antisymmetric function $(x \wedge y)\left(t_{1}, t_{2}\right)$ :

$$
\begin{aligned}
\left(A \wedge A-\lambda_{i} \lambda_{j} I \wedge I\right) x \wedge y & =A x \wedge A y-\lambda_{i} \lambda_{j} x \wedge y \\
& =A x \wedge A y-\lambda_{i} x \wedge A y+\lambda_{i} x \wedge A y-\lambda_{i} \lambda_{j} x \wedge y \\
& \text { [because of the linearity of the exterior product] } \\
& =\left(A x-\lambda_{i} x\right) \wedge A y+\lambda_{i} x \wedge\left(A y-\lambda_{j} y\right)=0 .
\end{aligned}
$$

From this we see that $\lambda_{i} \lambda_{j} \in \sigma_{p}(A \wedge A)$.

However, just as in the case of the tensor square of an operator, in order to obtain a statement, analogous to the finite-dimensional case, we need the additional assumption about complete continuity. For nonzero eigenvalues of the exterior square of a complete continuous operator, the following statement holds.

Theorem 5.2. Let $X$ be either $L_{p}(\Omega)$ or $C(\Omega)$ and let $\left\{\lambda_{i}\right\}$ be the set of all eigenvalues of an absolutely continuous operator $A: X \rightarrow X$, repeated according to multiplicity. Then all the possible products of the type $\left\{\lambda_{i} \lambda_{j}\right\}$, where $i<j$, form the set of all the possible (except, probably, zero) eigenvalues of the exterior square $A \wedge A$, repeated according to multiplicity.

Proof. The inclusion $\left\{\lambda_{i} \lambda_{j}\right\}_{i<j} \subset \sigma_{p}(A \wedge A)$, that is, each product of the form $\lambda_{i} \lambda_{j}$, where $i<j$, is an eigenvalue of $A \wedge A$, comes out from Theorem 5.1.

Now we will prove the reverse inclusion: $\sigma_{p}(A \wedge A) \subset\left\{\lambda_{i} \lambda_{j}\right\}_{i<j}$. As it was shown above in formulae (4.7) and (5.2),

$$
\sigma_{p}(A \wedge A) \backslash\{0\} \subset \sigma_{p}(A \otimes A) \backslash\{0\}=\sigma_{p}(A) \sigma_{p}(A) \backslash\{0\},
$$


that is, the operator $A \wedge A$ has no other eigenvalues, except products of the form $\lambda_{i} \lambda_{j}$. Enumerate the set of pairs $\{(i, j)\} i, j=1,2, \ldots$. In this way we get a numeration of $\left\{\lambda_{i} \lambda_{j}\right\}$ - the set of all eigenvalues of $A \otimes A$, repeated according to multiplicity. Decompose the obtained finite or countable set of indices $\Lambda$ in the following way:

$$
\Lambda=\Lambda_{1} \cup \Lambda_{2} \cup \Lambda_{3}
$$

where the set $\Lambda_{1}$ includes the numbers of those pairs $(i, j)$ for which $i<j, \Lambda_{2}$ includes those pairs for which $i=j$, and $\Lambda_{3}$ includes those pairs for which $i>j$. The set of all eigenvalues of $A \otimes A$, repeated according to multiplicity, will be then decomposed into three parts:

$$
\left\{\lambda_{\alpha}\right\}_{\alpha \in \Lambda}=\left\{\lambda_{\alpha}\right\}_{\alpha \in \Lambda_{1}} \cup\left\{\lambda_{\alpha}\right\}_{\alpha \in \Lambda_{2}} \cup\left\{\lambda_{\alpha}\right\}_{\alpha \in \Lambda_{3}} .
$$

As it was shown in Section 3, the operator $A \otimes A$ has a block structure, and so $\sigma_{p}(A \otimes A)$ can be decomposed into two subsets:

$$
\sigma_{p}(A \otimes A)=\sigma_{p}(A \wedge A) \cup \sigma_{p}\left(\left.A \otimes A\right|_{X^{s}}\right),
$$

where $X^{s}$ is the subset of all symmetric functions from $X$. In order to prove that the eigenvalues of $A \otimes A$, belonging to the sets $\left\{\lambda_{\alpha}\right\}_{\alpha \in \Lambda_{2}}$ and $\left\{\lambda_{\alpha}\right\}_{\alpha \in \Lambda_{3}}$, will not be the eigenvalues of $A \wedge A$, it is enough to show that they will be the eigenvalues of $\left.(A \otimes A)\right|_{X^{s}}$. Indeed, let $x_{i}(t) \in X$ be an eigenfunction of the operator $A$, corresponding to the eigenvalue $\lambda_{i}$. Let us examine the value of the operator $\left(A \otimes A-\lambda_{i}^{2} I \wedge I\right)$ on the function $x_{i}\left(t_{1}\right) x_{i}\left(t_{2}\right) \in X^{s}(\Omega \times \Omega)$ :

$$
\begin{aligned}
(A \otimes & \left.A-\lambda_{i}^{2} I \otimes I\right) x_{i}\left(t_{1}\right) x_{i}\left(t_{2}\right) \\
& =A x_{i}\left(t_{1}\right) A x_{i}\left(t_{2}\right)-\lambda_{i}^{2} x_{i}\left(t_{1}\right) x_{i}\left(t_{2}\right) \\
& =A x_{i}\left(t_{1}\right) A x_{i}\left(t_{2}\right)-\lambda_{i} x_{i}\left(t_{1}\right) A x_{i}\left(t_{2}\right)+\lambda_{i} x_{i}\left(t_{1}\right) A x_{i}\left(t_{2}\right)-\lambda_{i}^{2} x_{i}\left(t_{1}\right) x_{i}\left(t_{2}\right) \\
& =\left(A x_{i}-\lambda_{i} x_{i}\right)\left(t_{1}\right) A x_{i}\left(t_{2}\right)+\lambda_{i} x_{i}\left(t_{1}\right)\left(A x_{i}-\lambda_{i} x_{i}\right)\left(t_{2}\right)=0 .
\end{aligned}
$$

From this we see that $\lambda_{i}^{2} \in \sigma_{p}\left(\left.(A \otimes A)\right|_{X^{s}}\right)$. In an analogous way we can prove that a product of the form $\lambda_{i} \lambda_{j}$ will also be an eigenvalue of $\left.(A \otimes A)\right|_{X^{s}}$ (with the corresponding symmetric function $\left.x_{i}\left(t_{1}\right) x_{j}\left(t_{2}\right)+x_{j}\left(t_{1}\right) x_{i}\left(t_{2}\right)\right)$.

It is obvious that the spectrum of the operator $\mathbf{a} \circ(A \otimes A)$ coincides with the spectrum of $A \wedge A$.

\section{Generalization of the Gantmacher-Krein theorems in the case of 2-totally-nonnegative operators in the spaces $L_{p}(\Omega)$ and $C(\Omega)$}

Let us prove some generalizations of the Gantmacher-Krein theorems in the case of operators in the spaces $L_{p}(\Omega)$ and $C(\Omega)$, using the Kreinn-Rutman theorem (see, e.g., [14]) about completely continuous operators leaving invariant an almost-reproducing cone $K$ in a Banach space (for such operators we have the following property of the spectral radius: $\left.\rho(A) \in \sigma_{p}(A)\right)$. 
Theorem 6.1. Let $X$ be either $L_{p}(\Omega)$ or $C(\Omega)$, and, respectively, let $\tilde{X}$ be either $L_{p}(W)$ or $C_{0}(W)$. Let a completely continuous operator $A: X \rightarrow X$ with $\rho(A)>0$ leave invariant an almost-reproducing cone $K$ in $X$ and there is only one eigenvalue on the spectral circle $\lambda=\rho(A)$. Let its exterior square $A \wedge A: \tilde{X} \rightarrow \tilde{X}$ leave invariant an almost-reproducing cone $\widetilde{K}$ in $\tilde{X}$, besides $\rho(A \wedge A)>0$, and there is also only one eigenvalue on the spectral circle $\lambda=$ $\rho\left(\wedge^{j} A\right)$. Then the operator $A$ has a positive eigenvalue $\lambda_{1}=\rho(A)$, and its second (according to the module) eigenvalue $\lambda_{2}$ is also positive.

Proof. Enumerate eigenvalues of a completely continuous operator $A$, repeated according to multiplicity, in order of decrease of their modules:

$$
\left|\lambda_{1}\right|<\left|\lambda_{2}\right|<\left|\lambda_{3}\right| \geq \cdots
$$

Applying the Kreĭn-Rutman theorem to $A$, we get $\lambda_{1}=\rho(A)>0$. Now applying the Krel̆nRutman theorem to the operator $A \wedge A$ (that obviously is also completely continuous), we get $\rho(A \wedge A) \in \sigma_{p}(A \wedge A)$.

As it follows from the statement of Theorem 5.2, the exterior square of the operator $A$ has no other nonzero eigenvalues, except all the possible products of the form $\lambda_{i} \lambda_{j}$, where $i<j$. So, we get a conclusion that $\rho(A \wedge A)>0$ can be represented in the form of the product $\lambda_{i} \lambda_{j}$ with some values of the indices $i, j, i<j$, and from the fact that eigenvalues are numbered in a decreasing order, it follows that $\rho(A \wedge A)=\lambda_{1} \lambda_{2}$. Therefore $\lambda_{2}=\rho(A \wedge A) / \lambda_{1}>0$.

To this end a nonnegative linear operator $A$ is called indecomposable (see [2]) if it does not have any invariant components. For a linear operator which is indecomposable and nonnegative with respect to the cone of nonnegative functions in $L_{p}(\Omega)(C(\Omega))$ and such that $\rho(A)>0$, the positiveness and the simplicity of the first eigenvalue $\rho(A)$ is proved, for example, in $[1,2,9,11,12]$. An indecomposable operator $A$ is called primitive if its peripheral spectrum consists of the single point $\rho(A)$ and is called imprimitive if its peripheral spectrum contains more than one point. For imprimitive operators that are nonnegative with respect to the cone of nonnegative functions in $L_{p}(\Omega)(C(\Omega))$, the invariance of the spectrum of the operator $A$ with respect to some rotation is proved in $[1,8]$. (In [1] an analogue of the classical Frobenius theorem on the general form of primitive and imprimitive matrices is proved for compact indecomposable integral operators. This statement holds also true for arbitrary, not necessarily integral, compact indecomposable operators.) Call a cone $K$ in $L_{p}(\Omega)(C(\Omega))$ assumed if an indecomposable, nonnegative with-respect-to-the-cone- $K$, and completely continuous operator $A$ has the properties, proved in [1], that is, $\rho(A)$ is a positive simple eigenvalue of $A$; and if $A$ has $h$ eigenvalues, equal in modulus to $\rho(A)$, then each of them is simple and they coincide with the $h$ th roots of $\rho(A)^{h}$. We will call the operator $A$ 2-totally nonnegative if $A$ and $A \wedge A$ are nonnegative with respect to some assumed cones $K$ and $\tilde{K}$ in $L_{p}(\Omega)(C(\Omega))$ and $L_{p}(W)$ $\left(C_{0}(W)\right.$, resp.) and primitive.

Let us prove a generalization of one of the statements of Kreln and Gantmakher in the case of 2-totally-nonnegative operators in the spaces $L_{p}(\Omega)$ and $C(\Omega)$. 
Theorem 6.2. Let $X$ be either $L_{p}(\Omega)$ or $C(\Omega)$, and, respectively, let $\tilde{X}$ be either $L_{p}(W)$ or $C_{0}(W)$. Let a completely continuous operator $A: X \rightarrow X$ with $\rho(A)>0$ be nonnegative with respect to an assumed cone $K$ in $X$ and indecomposable, and let the operator $A \wedge A$ : $\tilde{X} \rightarrow \tilde{X}$ with $\rho(A \wedge A)>0$ be nonnegative with respect to an assumed cone $\widetilde{K}$ in $\tilde{X}$ and also indecomposable. Let $h(A)$ and $h(A \wedge A)$ be the indices of imprimitivity of $A$ and $A \wedge A$ respectively. Then

(a) either $h(A)=1$ and $h(A \wedge A)$ is arbitrary, or $h(A)=3$ and $h(A \wedge A)=3$;

(b) if $h(A)=1$ then the operator $A$ has two possible simple eigenvalues $\lambda_{1}, \lambda_{2}$, with

$$
\rho(A)=\lambda_{1}>\lambda_{2} \geq\left|\lambda_{3}\right| \geq \cdots ;
$$

(c) if $h(A)=h(A \wedge A)=1$, then $\lambda_{2}$ is different according to the module from the other eigenvalues;

(d) if $h(A)=1$ and $h(A \wedge A)>1$, then the operator $A$ has $h(A \wedge A)$ eigenvalues $\lambda_{2}$, $\lambda_{3}, \ldots, \lambda_{h(A \wedge A)+1}$, equal in modulus to $\lambda_{2}$, each of them is simple, and they coincide with the $h(A \wedge A)$ th roots of $\lambda_{2}^{h(A \wedge A)}$.

Proof. (a) First we will prove that if a completely continuous nonnegative operator $A$ is imprimitive with $h(A)=2$, then its exterior square can not be nonnegative. Really, according to the theorem of indecomposable operators, we have that there are two eigenvalues $\rho(A)>0$ and $-\rho(A)$ on the spectral circle of the operator $A$. As it follows, there is only one negative eigenvalue $-\rho^{2}(A)$ on the spectral circle of $A \wedge A$ and that is impossible if $A \wedge A$ is nonnegative.

Let $h(A)>3$ and let $A \wedge A$ be nonnegative. Prove that $A \wedge A$ is decomposable (i.e., it has invariant components). Suppose the opposite: let $A \wedge A$ be indecomposable. Then $\rho(A \wedge A)=\rho(A)^{2}$ and all the other eigenvalues on the spectral circle of $A \wedge A$ are simple. On the other hand, from Theorem 5.2 and imprimitivity of $A$, it follows that all the eigenvalues of $A \wedge A$, situated on the spectral circle, can be represented as couple products of different $h(A)$ th roots of $\rho(A)^{h(A)}$. Let us examine $\lambda_{j}=\rho(A) e^{2 \pi(j-1) i / h(A)}(j=$ $1, \ldots, h(A))$-all the eigenvalues of $A$, situated on the spectral circle. It is obvious that $\lambda_{2} \lambda_{h(A)}=\lambda_{3} \lambda_{h(A)-1}=\cdots=\lambda_{k} \lambda_{h(A)-(k-2)}=\cdots=\rho(A)^{2}$. As it follows, $\rho(A \wedge A)=\rho(A)^{2}$ is not a simple eigenvalue of $A \wedge A$.

Prove that if $A$ is imprimitive with its index $h(A)=3$ and its exterior square is indecomposable, then $A \wedge A$ is also imprimitive with $h(A \wedge A)=3$. Indeed, in this case there are three eigenvalues $\lambda_{1}=\rho(A), \lambda_{2}=\rho(A) e^{2 \pi i / 3}, \lambda_{3}=\rho(A) e^{4 \pi i / 3}$ on the spectral circle of the operator $A$ and there are also three eigenvalues $\lambda_{1} \lambda_{2}=\rho(A)^{2} e^{2 \pi i / 3}, \lambda_{1} \lambda_{3}=\rho(A)^{2} e^{4 \pi i / 3}$, and $\lambda_{2} \lambda_{3}=\rho(A) e^{2 \pi i / 3} \rho(A) e^{4 \pi i / 3}=\rho(A)^{2}$ that coincide with the 3rd roots of $\left(\rho(A)^{2}\right)^{3}$, on the spectral circle of $A \wedge A$.

(b) The existence and the positiveness of the first and the second eigenvalues follow from Theorem 6.1. The simplicity of $\lambda_{2}$ follows from the equality $\lambda_{2}=\rho(A \wedge A) / \rho(A)$ and the simplicity of eigenvalues $\rho(A)$ and $\rho(A \wedge A)$.

(c) In the case of $h(A)=h(A \wedge A)=1$ the distinction according to the module of $\lambda_{2}$ from other eigenvalues is obvious. 
(d) In case of $h(A \wedge A)>1$ from Theorem 5.2 and the properties of the peripheral spectrum of the imprimitive operator $A \wedge A$ it follows that for an eigenvalue $\lambda_{j}, j=$ $2, \ldots, h(A \wedge A)+1$ the next equality holds: $\lambda_{j}=\rho(A \wedge A) e^{2 \pi(j-1) i / h(A \wedge A)} / \rho(A)$.

Consider an example of operator for which the conditions of Theorem 6.2 are satisfied and $h(A)=3$. Let the operator $A: \mathbb{R}^{3} \rightarrow \mathbb{R}^{3}$ be defined by the matrix

$$
\mathbf{A}=\left(\begin{array}{lll}
0 & 0 & 1 \\
1 & 0 & 0 \\
0 & 1 & 0
\end{array}\right)
$$

It is obvious that this operator is nonnegative with respect to the cone of nonnegative vectors in $\mathbb{R}^{3}$ and imprimitive with $h(A)=3$. In the basis, which consists of exterior products of the given basic vectors, the matrix of the exterior square of operator $A \wedge A$ coincides with the second associated matrix, that is, it can be represented in the following form:

$$
\mathbf{A} \wedge \mathbf{A}=\left(\begin{array}{ccc}
0 & -1 & 0 \\
0 & 0 & -1 \\
1 & 0 & 0
\end{array}\right)
$$

It is obvious that $A \wedge A$ is imprimitive with $h(A \wedge A)=3$. It is also obvious that it leaves invariant the cone of vectors $(1,0,0),(0,-1,0)$, and $(0,0,1)$.

Given an operator $A$ in the finite-dimensional space $\mathbb{R}^{3}$, it is not difficult, using the standard scheme, to define a linear integral operator with the same properties, acting in $L_{p}(\Omega)$ or $C(\Omega)$.

\section{Linear integral operators in the spaces $L_{p}(\Omega)$ and $C(\Omega)$}

Let us examine a linear integral operator $A$ with kernel $k(t, s)$, acting in the space $L_{p}(\Omega)$. Observing that

$$
\begin{aligned}
(A \otimes A) x\left(t_{1}, t_{2}\right) & =A_{(1)} A_{(2)} x\left(t_{1}, t_{2}\right) \\
& =\int_{\Omega} k\left(t_{1}, s_{1}\right)\left(\int_{\Omega} k\left(t_{2}, s_{2}\right) x\left(s_{1}, s_{2}\right) d \mu\right) d \mu \\
& =\int_{\Omega} \int_{\Omega} k\left(t_{1}, s_{1}\right) k\left(t_{2}, s_{2}\right) x\left(s_{1}, s_{2}\right) d(\mu \otimes \mu)\left(s_{1}, s_{2}\right),
\end{aligned}
$$

we conclude that the tensor square of the operator $A$ is a linear integral operator with kernel $k\left(t_{1}, s_{1}\right) k\left(t_{2}, s_{2}\right)$, acting in the space $L_{p}(\Omega \times \Omega)$. Thus the exterior square of $A$ is a restriction of the integral operator with kernel $k\left(t_{1}, s_{1}\right) k\left(t_{2}, s_{2}\right)$ onto the subspace $L_{p}^{a}(\Omega \times \Omega)$. 
Let us examine the value of the operator $\mathbf{a} \circ(A \otimes A)$ on an arbitrary function $x\left(t_{1}, t_{2}\right)$ from $L_{p}(\Omega \times \Omega)$ :

$$
\begin{aligned}
& \mathbf{a} \circ\left(\int_{\Omega \times \Omega} k\left(t_{1}, s_{1}\right) k\left(t_{2}, s_{2}\right) x\left(s_{1}, s_{2}\right) d(\mu \otimes \mu)\left(s_{1}, s_{2}\right)\right) \\
& \quad=\frac{1}{2} \int_{\Omega \times \Omega} k\left(t_{1}, s_{1}\right) k\left(t_{2}, s_{2}\right) x\left(s_{1}, s_{2}\right) d(\mu \otimes \mu)\left(s_{1}, s_{2}\right) \\
& \quad-\frac{1}{2} \int_{\Omega \times \Omega} k\left(t_{2}, s_{1}\right) k\left(t_{1}, s_{2}\right) x\left(s_{1}, s_{2}\right) d(\mu \otimes \mu)\left(s_{1}, s_{2}\right) \\
& =\frac{1}{2} \int_{\Omega \times \Omega}\left|\begin{array}{ll}
k\left(t_{1}, s_{1}\right) & k\left(t_{1}, s_{2}\right) \\
k\left(t_{2}, s_{1}\right) & k\left(t_{2}, s_{2}\right)
\end{array}\right| x\left(s_{1}, s_{2}\right) d(\mu \otimes \mu)\left(s_{1}, s_{2}\right) .
\end{aligned}
$$

Since the values of the operators $\mathbf{a} \circ(A \otimes A)$ and $A \wedge A$ on antisymmetric functions do coincide, it is obvious that

$$
\begin{aligned}
\int_{\Omega \times \Omega} & k\left(t_{1}, s_{1}\right) k\left(t_{2}, s_{2}\right) x\left(s_{1}, s_{2}\right) d(\mu \otimes \mu)\left(s_{1}, s_{2}\right) \\
= & \mathbf{a} \circ \int_{\Omega \times \Omega} k\left(t_{1}, s_{1}\right) k\left(t_{2}, s_{2}\right) x\left(s_{1}, s_{2}\right) d(\mu \otimes \mu)\left(s_{1}, s_{2}\right) \\
= & \frac{1}{2} \int_{\Omega \times \Omega}\left|\begin{array}{ll}
k\left(t_{1}, s_{1}\right) & k\left(t_{1}, s_{2}\right) \\
k\left(t_{2}, s_{1}\right) & k\left(t_{2}, s_{2}\right)
\end{array}\right| x\left(s_{1}, s_{2}\right) d(\mu \otimes \mu)\left(s_{1}, s_{2}\right),
\end{aligned}
$$

where $x\left(t_{1}, t_{2}\right)$ is an arbitrary function from $L_{p}^{a}(\Omega \times \Omega)$.

Further, we will call the kernel $\left|\begin{array}{lll}k\left(t_{1}, s_{1}\right) & k\left(t_{1}, s_{2}\right) \\ k\left(t_{2}, s_{1}\right) & k\left(t_{2}, s_{2}\right)\end{array}\right|$ the second associated kernel $k(t, s)$ and will denote it by $(k \wedge k)\left(t_{1}, t_{2}, s_{1}, s_{2}\right)$.

Since $\Omega \times \Omega=W \cup \widetilde{W}$, we have that

$$
\begin{aligned}
(A \wedge A) & x\left(t_{1}, t_{2}\right) \\
= & \frac{1}{2} \int_{W}(k \wedge k)\left(t_{1}, t_{2}, s_{1}, s_{2}\right) x\left(s_{1}, s_{2}\right) d(\mu \otimes \mu)\left(s_{1}, s_{2}\right) \\
& +\frac{1}{2} \int_{\widetilde{W}}(k \wedge k)\left(t_{1}, t_{2}, s_{1}, s_{2}\right) x\left(s_{1}, s_{2}\right) d(\mu \otimes \mu)\left(s_{1}, s_{2}\right) \\
= & \cdots
\end{aligned}
$$

Exchanging the places of $s_{1}$ and $s_{2}$ in the second integral, we get

$$
\cdots=\int_{W}(k \wedge k)\left(t_{1}, t_{2}, s_{1}, s_{2}\right) x\left(s_{1}, s_{2}\right) d(\mu \otimes \mu)\left(s_{1}, s_{2}\right) .
$$

Now we can consider the exterior square of the operator $A$, acting in the space $L_{p}(\Omega)$, as a linear integral operator, acting in $L_{p}(W)$, with the kernel equal to the second associated to $k(t, s)$. The same reasoning is true for the space $C(\Omega)$.

A nonnegative kernel $k(t, s)$ is called indecomposable if for any measurable set $D \in$ $\Omega, 0<\mu(D)<\mu(\Omega)$, there exist measurable sets $A \in D, B \in \Omega \backslash D$, such that $\mu(A)>0$, 
$\mu(B)>0$ and $k(t, s)>0$ almost everywhere on $A \times B$. A nonnegative integral operator $A$ with a kernel $k(t, s)$ is indecomposable if and only if the kernel $k(t, s)$ is indecomposable (see [8]). In [1], for an indecomposable nonnegative linear integral operator, the positiveness and the simplicity of the eigenvalue $\rho(A)$ is proved (see $[1$, page 6 , Theorem 2]).

An indecomposable kernel $k(t, s)$ is called imprimitive, if there exists a decomposition of the set $\Omega$ in $n>1$ nonintersecting sets $\Omega_{j}(j=1, \ldots, n)$ of a positive measure: $\Omega=$ $\cup_{j=1}^{n} \Omega_{j}$, for which $k(t, s)=0$ with $t \in \Omega_{j}, s \notin \Omega_{j+1}$ for any $j=1, \ldots, n$ (in the case of $j=n$ we presuppose $j+1$ to be equal to 1$)$. Otherwise the kernel $k(t, s)$ is called primitive. A compact integral operator $A$ is imprimitive (resp., primitive) if and only if its kernel is imprimitive (primitive). A kernel $k(t, s)$ is called 2-totally nonnegative, if both $k(t, s)$ and $(k \wedge k)\left(t_{1}, t_{2}, s_{1}, s_{2}\right)$ are primitive. Further, we will examine on the set $W$ the second associated kernel and the conditions implied to it. The index of imprimitivity of $(k \wedge$ $k)\left(t_{1}, t_{2}, s_{1}, s_{2}\right)$ will be denoted by $h(k \wedge k)$.

Let the kernel $k(t, s)$ of a completely continuous integral operator $A$ in the space $L_{p}(\Omega)$ or $C(\Omega)$ be nonnegative and primitive. Let its second associated kernel be also nonnegative and primitive. Then Theorem 6.2 implies that the second, according to the module, eigenvalue of the operator $A \lambda_{2}$ is positive, simple, and different in modulus from the other eigenvalues.

Note that in this reasoning the kernel is not presupposed to be continuous, but only assume that the operator $A$ acts in the space $L_{p}(\Omega)$ or $C(\Omega)$.

\section{Concluding remarks}

All the results given in the present paper can be easily spread on $k$-totally-nonnegative operators $(k>2)$, acting in the space $L_{p}(\Omega)$ or $C(\Omega)$. Similar results will be also true for other spaces, for example, for some ideal spaces, the Lorenz-Martzinkevich space, and so forth.

\section{References}

[1] E. A. Alekhno and P. P. Zabreiko, Quasipositive elements and indecomposable operators in ideal spaces. II, Vestsī Natsyyanal'nă Akadèmīī Navuk Belarusī. Seryya Fīzīka-Matèmatychnykh Navuk (2003), no. 1, 5-10, 124.

[2] T. Andô, Positive linear operators in semi-ordered linear spaces, Journal of the Faculty of Science, Hokkaido University. Series I. 13 (1957), 214-228.

[3] F. R. Gantmacher and M. G. Krě̆n, Oszillationsmatrizen, Oszillationskerne und kleine Schwingungen mechanischer Systeme, Wissenschaftliche Bearbeitung der deutschen Ausgabe: Alfred Stöhr. Mathematische Lehrbücher und Monographien, I. Abteilung, vol. 5, Akademie, Berlin, 1960.

[4] T. Ichinose, Operators on tensor products of Banach spaces, Transactions of the American Mathematical Society 170 (1972), 197-219.

[5] - Operational calculus for tensor products of linear operators in Banach spaces, Hokkaido Mathematical Journal 4 (1975), no. 2, 306-334.

[6] Spectral properties of tensor products of linear operators. I, Transactions of the American Mathematical Society 235 (1978), 75-113. 
[7] __ Spectral properties of tensor products of linear operators. II. The approximate point spectrum and Kato essential spectrum, Transactions of the American Mathematical Society 237 (1978), 223-254.

[8] M. A. Krasnosel'skij, Je. A. Lifshits, and A. V. Sobolev, Positive Linear Systems. The Method of Positive Operators, Sigma Series in Applied Mathematics, vol. 5, Heldermann, Berlin, 1989.

[9] H. P. Lotz, Über das Spektrum positiver Operatoren, Mathematische Zeitschrift 108 (1968), no. 1, $15-32$.

[10] T.-W. Ma, Classical Analysis on Normed Spaces, World Scientific, New Jersey, 1995.

[11] F. Niiro and I. Sawashima, On the spectral properties of positive irreducible operators in an arbitrary Banach lattice and problems of $H$. H. Schaefer, Scientific Papers of the College of Arts and Sciences. The University of Tokyo 16 (1966), 145-183.

[12] I. Sawashima, On spectral properties of some positive operators, Natural Science Report of the Ochanomizu University 15 (1964), 53-64.

[13] C. Stéphanos, Sur une extension du calculus des substitutions linéaires, Journal de Mathematiques Pures et Appliquees (5) 6 (1900), 73-128.

[14] P. P. Zabreiko and S. V. Smitskikh, A theorem of M. G. Krein and M. A. Rutman, Functional Analysis and Its Applications 13 (1980), 222-223.

O. Y. Kushel: Mechanics and Mathematics Faculty, Belarusian State University, Pr. Independence 4, 220050 Minsk, Belarus

E-mail address: kushel@mail.ru

P. P. Zabreiko: Mechanics and Mathematics Faculty, Belarusian State University, Pr. Independence 4, 220050 Minsk, Belarus

E-mail address: zabreiko@bsu.by 


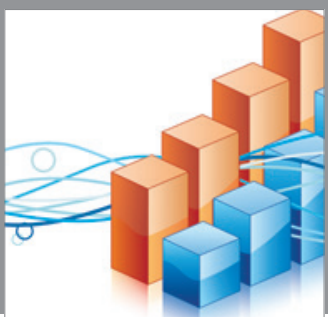

Advances in

Operations Research

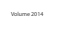

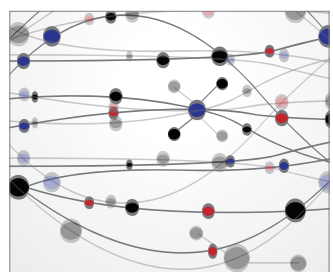

\section{The Scientific} World Journal
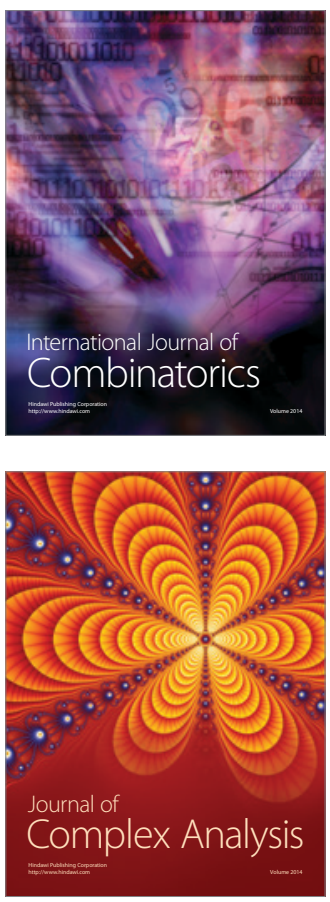

International Journal of

Mathematics and

Mathematical

Sciences
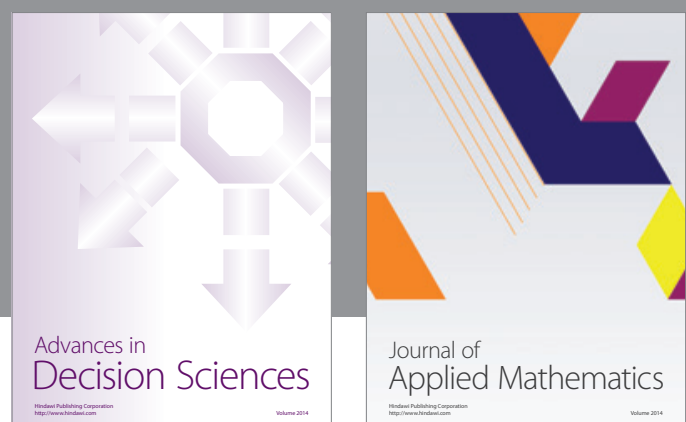

Journal of

Applied Mathematics
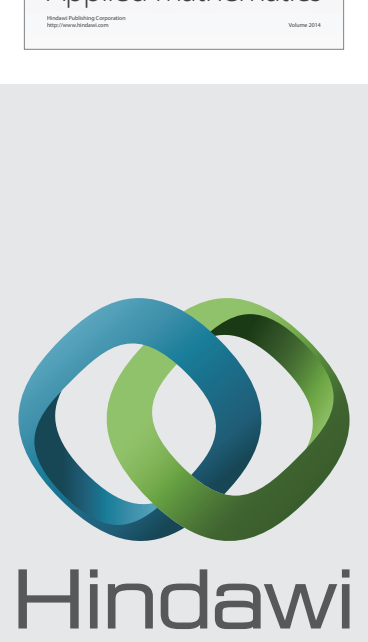

Submit your manuscripts at http://www.hindawi.com
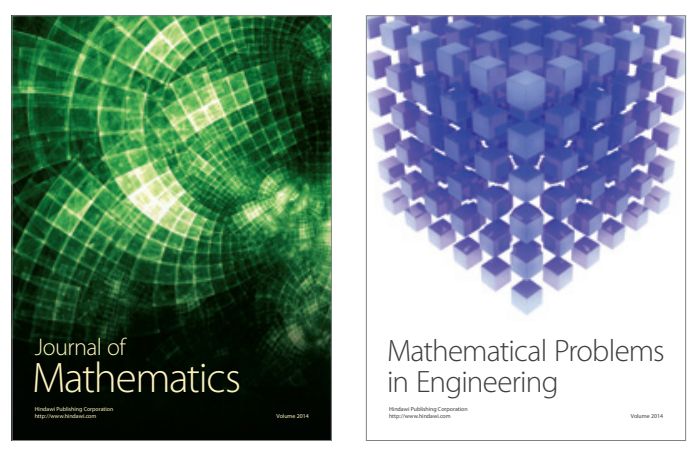

Mathematical Problems in Engineering
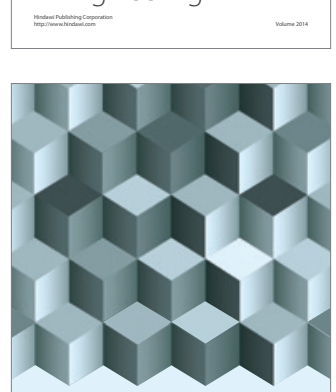

Journal of

Function Spaces
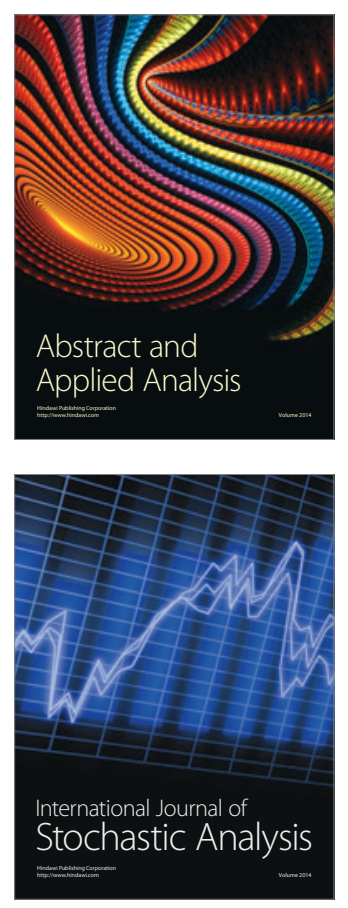

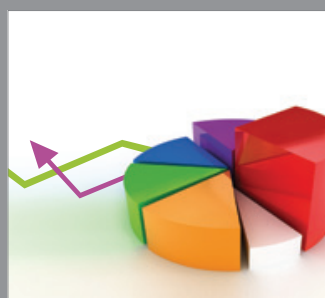

ournal of

Probability and Statistics

Promensencen
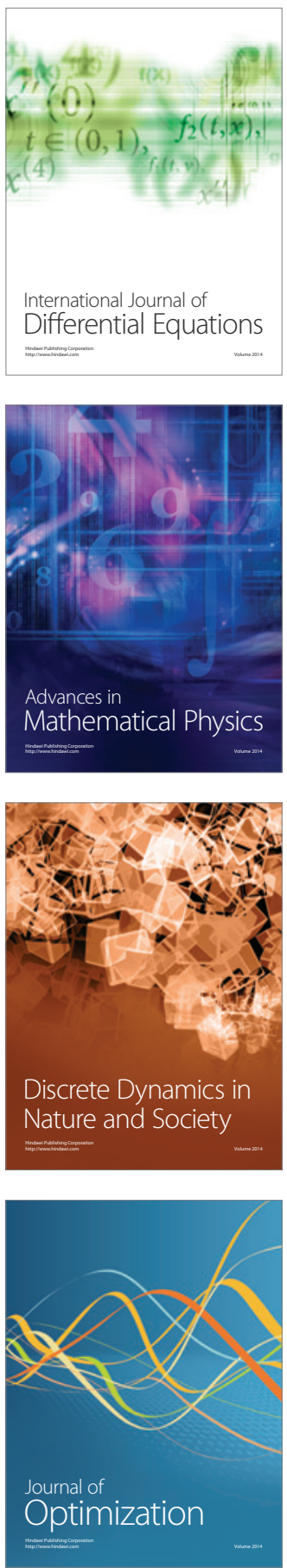